Klaus Dörre/ Oliver Nachtwey

\title{
Auf dem Weg zur Erneuerung - Organizing als politisches Instrument
}

Schwindende gewerkschaftliche Organisationsmacht wird in den Sozialwissenschaften seit langem thematisiert (Dribbusch 2003). Allein zwischen 1993 und 2003 haben die europäischen Gewerkschaften im Durchschnitt 15 Prozent ihrer Mitglieder verloren. Die DGB-Gewerkschaften machen keine Ausnahme. Gegenwärtig entspricht ihre absolute Mitgliederzahl in Gesamtdeutschland nur noch jener der West-Gewerkschaften von 1969/70. Sicher gibt es in jüngster Zeit auch positive Gegentendenzen. So hat die IG Metall 2008 erstmals seit vielen Jahren den Mitgliederrückgang stoppen können. Vor allem bei jüngeren Beschäftigten konnte sie erheblich zulegen (Infodienst 2009). Ob man deshalb bereits von einem ,Comeback der Gewerkschaften" (Tagesspiegel, 01.02.2009) sprechen kann, bleibt abzuwarten. Dass die Möglichkeit einer Revitalisierung von Gewerkschaften überhaupt wissenschaftlich ausgeleuchtet wird, ist ein Verdienst der so genannten ,Labour Revitalization Studies“ (Frege/Kelly 2004). In Kontrast zum fatalistischen Tenor vieler zeitgenössischer Analysen lenken sie das Augenmerk auf die strategische Wahl, auf die Möglichkeiten von Gewerkschaften, kreativ zu handeln und sich selbst zu erneuern.

Anhand wichtiger Charakteristika lässt sich zeigen, dass manche deutsche Gewerkschaftsgliederungen gewissermaßen aus der Not geboren und teilweise ohne expliziten Bezug auf Vorbilder aus Übersee zumindest informell bereits manches von dem praktizieren, was in der Literatur am Beispiel amerikanischer Organizing-Gewerkschaften als Innovation diskutiert wird. Bewegungsorientierung, Mitgliederpartizipation und Kampagnenfähigkeit bezeichnen einen Möglichkeitsraum, innerhalb dessen eine strategische Wahl von Gewerkschaften er- folgen kann. Die Schwierigkeiten, OrganizingKonzepte erfolgreich zu praktizieren, resultieren nicht allein aus Besonderheiten des jeweiligen Arbeitsbeziehungs-Systems. Vielmehr, so die These, stehen Gewerkschaften sowohl in angelsächsisch-voluntaristischen als auch in korporativen Systemen vor der Herausforderung, neue Machtquellen jenseits angestammter Machtressourcen erschließen zu müssen. Nicht die Adaption einzelner Organizing-Praktiken, sondern deren Verknüpfung und Verstetigung in einem kohärenten Konzept assoziierter Macht erweist sich als eigentliches Problem gewerkschaftlicher Erneuerungsstrategien.

\section{Organizing - Made in USA}

Gewerkschaftliche Handlungsstrategien variieren, wenn die Institutionensysteme unter Veränderungsdruck geraten. Institutionalisierte Arbeitsbeziehungen legen den Gewerkschaften Handlungsstrategien nahe, die auch dann noch überzeugend erscheinen, wenn sich gesellschaftliche Kräfteverhältnisse bereits gravierend verändert haben. Nun darf die Erosion institutioneller Macht, wie sie sich in vielen Metropolenkapitalismen abzeichnet, nicht mit einem generellen Verschwinden von Arbeitermacht gleichgesetzt werden. Beverly Silver (2005) hat in ihrer eindrucksvollen Studie gezeigt, wie geographische Verlagerungen von Produktionsstandorten neue raum-zeitliche, Fixierungen " von Kapital und mit ihnen neue Arbeiterklassen und Arbeiterbewegungen an den jeweils bevorzugten Produktionsstandorten hervorbringen. Die Autorin skizziert eine räumliche Diffusion von Produktionsmacht, welche sich in vielen Ländern des Südens zunächst in , labour unrest ', in spontanen Unruhen und Aufständen äußert. Es 
finden sich aber auch Beispiele für eine Revitalisierung gewerkschaftlicher Organisationsmacht - und das nicht nur in sich entwickelnden Ländern wie Brasilien, Südkorea oder Südafrika, sondern auch in Zentrumsstaaten wie den USA.

Stilbildend für die internationale Debatte um gewerkschaftliche Emeuerung ist das Beispiel der Service Employees International Union (SEIU) und ihrer inzwischen legendären Kampagne ,Justice for Janitors'. Die SEIU hat sich infolge eines umfassenden Organisationswandels innerhalb kurzer Zeit von einer dahinsiechenden Business Union in eine konfliktfähige Gewerkschaft mit den höchsten Mitgliederzuwächsen in den USA gemausert. Andere nordamerikanische Gewerkschaften wie UNITE (Union of Needletrades, Industrial and Textile Employees) HERE (Hotel Employees and Restaurant Employees International Union), UBC (Union Brotherhood of Carpenters and Joiners of America) und LIUNA (Laborers' International Union of North America) haben sich inzwischen ebenfalls für eine Ausrichtung am sogenannten ,Organizing-Modell' entschieden. Markenzeichen der genannten Gewerkschaften ist, dass sie sämtliche Aktivitäten mit dem Ziel einer nachhaltigen Stärkung ihrer Organisationsmacht verbinden. Und just diese Fokussierung dürfte wohl erheblich zur raschen internationalen Rezeption des ,Organizing-Modells * beigetragen haben.

Die Redeweise von einem Modell suggeriert indessen eine begrifflich-konzeptionelle Eindeutigkeit, die so nicht existiert. Gegenwärtig wird unter gewerkschaftlichen PraktikerInnen und WissenschaftlerInnen kontrovers diskutiert, welche Handlungsstrategien, Organisationsformen und interessenpolitischen Inhalte mit dieser Organizing-Kategorie bezeichnet werden können. In der deutschen Debatte werden teilweise Definitionen favorisiert, die sich wesentlich auf Organisationstechniken und Methoden der Mitgliederwerbung konzentrie- ren (z. B. Dribbusch 2007, S. 30 ff.). Das ist durchaus legitim, zumal Fragen nach der Übertragbarkeit von Organizing-Ansätzen auf deutsche Verhältnisse sich auf diese Weise pragmatisch beantworten lassen. Allerdings koppelt ein solch enges Verständnis die Organizing-Debatte von der inhaltlichen Dimension gewerkschaftlicher Erneuerung ab. Um dies zu vermeiden, orientieren wir uns an einem weiten, eher analytischen Organzing-Verständnis, wie es u. a. Voss und Sherman (2000) vorgeschlagen haben. In ihrem Klassiker der Labor Revitalization Studies zeichnen sie nach, wie bürokratische Strukturen und eingeschliffene Praktiken so verändert werden können, dass es zu einer Wiederbelebung gewerkschaftlicher Aktivitäten und zu einer erhöhten Mitgliederbindung kommt. Entscheidend ist demnach der Wechsel von einem Service-Modell, das eine weitgehend passive Mitgliedschaft über qualifizierte Dienstleistungen zu binden sucht, zu einem Organizing-Modell, das auf Mitgliedermobilisierung setzt, die Arbeitspraxis der Basisorganisationen verändert und neue, partizipative Organisationsstrukturen schafft.

Von Beispielen aus den USA inspiriert, arbeiten Voss/Sherman (2000) drei Faktorenbündel heraus, die den erfolgreichen Organisationswandel erklären sollen. Zum einen schafft das verbreitete Bewusstsein über eine tiefe politische Krise der Organisation an der Basis Voraussetzungen für einen Führungswechsel. Das neue Personal, das sich lokal durchsetzt, verbindet die eigene Positionierung mit einem Strategiewechsel. Zum anderen bleibt der Wandel aber nicht auf die örtlichen Gewerkschaftsgliederungen beschränkt. Er erfasst die gesamte nationale Organisation und ist, wie im Fall der SEIU, teilweise mit heftigen Konflikten an der Spitze der Gewerkschaft verbunden. Von zentraler Bedeutung ist indessen eine dritte Beobachtung: Ein Teil der Führungskräfte, die mitunter aufgrund von Druck aus der Zentrale neu eingestellt werden, kommt, von außen' und 
verfügt über Erfahrungen mit sozialen Bewegungen, Graswurzelinitiativen und Stadtteilarbeit. Den bewegungssozialisierten Gewerkschaftern fällt es offenbar leichter, einen umfassenden Strategiewechsel in Gang zu setzen. Sie verfügen in der Regel über komplexere Gerechtigkeitsvorstellungen als Gewerkschafter, mit Stallgeruch'. Eingefahrene Gewerkschaftstraditionen und Handlungsroutinen verfangen nicht. Dafür sind die neuen Aktivisten mit Techniken sozialer Mobilisierung in der Zivilgesellschaft bestens vertraut. Aufgrund ihrer alten Kontakte sehen sie sich in der Lage, Bündnisbeziehungen zu NGOs und Bewegungen auBerhalb der Arbeitswelt zu knüpfen, um deren besondere Machtressourcen für die Gewerkschaft zugänglich zu machen.

Schon die starke Betonung des Bewegungsmoments signalisiert, dass Voss/Sherman mit dem Organizing-Modell keineswegs ein inhaltsleeres Set an Rekrutierungstechniken assoziieren. Vielmehr beschreiben sie einen Typus des Gewerkschaftshandelns, der sich durch die Beteiligung von Mitgliedern an der Politikentwicklung, durch unkonventionelle und teilweise höchst konfliktträchtige Aktionsformen sowie eine thematisch breite politische Agenda auszeichnet. Im Zentrum offensiver Organisierungsmodelle steht die Gewinnung neuer Mitglieder, ein Ziel, zu dessen Realisierung unkonventionelle, teilweise auch konfrontative Taktiken angewendet werden (ebd., S. 316). Charakteristisch für entsprechende Ansätze ist die starke Betonung von sozialer Gerechtigkeit und Menschenwürde. So verstanden, impliziert der Übergang zum Organizing-Modell einen strategischen Organisationswandel, der auch die Beziehungen zwischen gewerkschaftlichen Funktionsträgern und (potenziellen) Mitgliedern nachhaltig verändert. Nachfolgend beschränken wir uns auf die Präsentationen einiger ausgewählter Forschungsergebnisse. Wir gehen davon aus, dass Bewegungsorientierung, Mitgliederpartizipation und Kampagnenfähigkeit ein konzeptionelles Dreieck bilden, in dem sich - jenseits institutioneller Besonderheiten der nationalen Systeme der industriellen Beziehungen - gewerkschaftliche Erneuerung vollziehen kann.

\section{Bewegungsorientierung}

Die inhaltliche Ausrichtung weit gefasster Organizing-Ansätze wird von einem Teil der wissenschaftlichen Interpreten (z. B. Frege 2000) unter der Bezeichnung Social Movement Unionism thematisiert. Damit ist gemeint, dass Gewerkschaften ihre Mobilisierungs- und Konfliktfähigkeit verbessern wollen, indem sie zentrale gesellschaftliche Themen in ihre politische Agenda integrieren. Ursprünglich war die Kategorie Bewegungsgewerkschaft auf neue Arbeiterbewegungen und erstarkende Gewerkschaften in einigen Ländern des Südens (Brinkmann u.a. 2008, S. 45-68) gemünzt. Diese schwach institutionalisierten Gewerkschaften setzen auf Massenmobilisierung, sie verfügen über eine entwickelte Beteiligungskultur in ihrer Mitgliedschaft, organisieren ihre Kämpfe über die Fabrikgrenzen hinaus und machen Bündnisse mit sozialen Bewegungen außerhalb der Arbeitssphäre zu einem konstitutiven Element ihres strategischen Handelns.

Offenkundig fällt es Gewerkschaften, die vornehmlich als bessere Problemlöser mit dem Management konkurrieren, schwer, organisationspolitisch erfolgreich zu sein, weil der Gewerkschaftsanteil an den ,Problemlösungen ' für die Beschäftigten schwer nachzuvollziehen ist und von solchen Ansätzen kein Beteiligungsimpuls ausgeht. (Cregan 2005). Insofern passen Interessenpolitiken, die Gerechtigkeitsfragen gegenüber dem Nachweis wirtschaftlicher Effizienz priorisieren (Aronowitz 2005), in vielen Fällen offenbar besser zum Anspruch offensiver Organizing-Ansätze (Fantasia/Voss 2004, S. 127-130) als wertschöpfungsorientierte Strategien. Auch weil Bewegungspolitiken sich nicht beliebig auf Dauer stellen lassen, ringen 
nicht nur die südlichen Bewegungs- sondern auch die nordamerikanischen, Organizing-Gewerkschaften' um eine Institutionalisierung ihrer Verhandlungsmacht. Festhalten lässt sich aber, dass Organizing-Ansätze in vielen Fällen in interessenpolitische Strategien eingebettet sind, die zentrale gesellschaftliche Themen und Konfliktfelder offensiv zu besetzen suchen.

\section{Mitgliederpartizipation}

Direkte Mitgliederbeteiligung ist für viele AutorInnen eine zentrale politisch-organisatorische Form des Organizing. Fiorito (2004) sieht in der Dezentralisierung von Entscheidungen, in offenen Strukturen, die eine Beteiligung bis hin $\mathrm{zu}$, Mitgliederselbstbestimmung ‘ erlauben, eine entscheidende Erfolgsbedingung offensiver Organizing-Konzepte. Dabei geht es nicht allein um die Verbreiterung der aktiven Basis einer Gewerkschaft. Die Beziehungen zwischen Aktivisten und ,normalen' Mitgliedern beruhen, wie Beaud/Pialoux (2004) in ihrer Studie über das Peugeot-Werk in Sochaux gezeigt haben, auf einem eingespielten ,System der Erwartungen und Errungenschaften" (ebd., S. 259), das aufgrund von Veränderungen im Arbeitsprozess und in der Zusammensetzung der Belegschaften periodisch erneuert werden muss. Ein solcher Erneuerungsbedarf existiert offenkundig, auch weil die Aktivisten angesichts knapper Ressourcen und fehlender Erfolge dazu tendieren, sich durch immer intensivere, Organisationsarbeit ' von den Repräsentierten zu entfernen. Empirische Forschungen haben belegt, dass die soziale Heterogenität potentieller Mitglieder nicht automatisch zu einem Verlust an gewerkschaftlicher Bindekraft führen muss. Vielmehr wird eine verbesserte Teilhabe der Mitglieder an Organisationsentscheidungen zur entscheidenden Einflussgröße. Innergewerkschaftliche Demokratie und reale Partizipationschancen, besitzen laut Lévesque u. a. (2005) die mit Abstand höchste Erklärungskraft bei der Abwehr von Mitgliederunzufriedenheit. Markowitz
(1999) zeigt darüber hinaus, dass die Stabilisierung partizipativer Beziehungen zwischen Funktionsträgern und neu gewonnenen Mitgliedern wichtig ist, um neue Gewerkschafter , after Organizing' in der Organisation zu halten. Innerorganisatorische Mitbestimmung gilt Autoren wie Clawson (2005) als Schlüssel für eine erfolgreiche Ansprache gewerkschaftlich bislang schwach repräsentierter Gruppen (Frauen, Prekäre, Minderheiten). Kommunikation, von Angesicht zu Angesicht' und Authentizität der aktiven Gewerkschafter (Vertretung durch Gleiche) sind demnach ausschlaggebend für nachhaltig veränderte Beziehungen zwischen Repräsentanten und Repräsentierten.

Sprechen solche Forschungsergebnisse für einen engen Zusammenhang zwischen Mitgliederpartizipation und organisatorisch-politischer Bindekraft der Gewerkschaften, bleibt doch offen, ob und wie sich ein beteiligungsorientierter Politikstil längerfristig durchhalten lässt. Aus der Bewegungsforschung ist bekannt, dass es nicht nur schwer, sondern geradezu unmöglich ist, ein hohes Partizipationsniveau von Mitgliedern über längere Zeiträume hinweg zu stabilisieren. Insofern werden sich auch die intelligentesten Organizing-Ansätze immer wieder mit dem Problem konfrontiert sehen, dass stärker partizipative und eher repräsentative Ansätze einander abwechseln und ergänzen müssen. Die Kunst besteht offenbar darin, Führungsfähigkeit und Partizipation optimal zu kombinieren.

\section{Kampagnenfähigkeit}

Eine strikte Kampagnenorientierung gilt vielen Autoren als geeignetes Mittel, um Bewegungsorientierung und Mitgliederpartizipation projektgebunden zu verstetigen. Aus den unterschiedlichen Kampagnenformen ragen die Comprehensive Campaigns heraus. Dabei handelt es sich um einen Kampagnentyp, in dessen Verlauf sich die Gewerkschaften selbst nachhaltig verändern. Bronfenbrenner/Hickey (2004) gelangen in ihrer Analyse zu dem überraschenden 
Ergebnis, dass positive Resultate nicht so sehr von der Branche oder der Unternehmensstruktur abhängen, sondern vor allem von Art und Intensität der Kampagne beeinflusst werden. Ein Großteil dessen, was die beiden Autoren als Essentials einer erfolgreichen Kampagnenorientierung benennen (ebd., S. 37-41), wirkt auf den ersten Blick wenig spektakulär. Das gilt etwa für die Forderung nach Trainingsmaßnahmen auch während der Kampagne oder die Festlegung von Zwischenzielen. Anderes wie z. B. die Implementierung von Kampagnen auf dezentraler Ebene, das Vermeiden innergewerkschaftlicher Organisationskonkurrenzen oder die internationale Bearbeitung von Themen in multinationalen Unternehmen erscheint nahe liegend, markiert aber doch einen deutlichen Kontrast zu gewerkschaftlichen Organisationsroutinen.

Insgesamt belegen einschlägige Studien (Gall 2005), dass eine Kampagnenorientierung höchst
Unterschiedliches bedeuten kann. Während sich die internen Hindernisse (fehlende OrganizingTradition, interner Widerstand, geringe Unterstützung durch die Zentrale) für erfolgreiches Organizing in vielen Ländern gleichen, gibt es bei den externen Blockierungen $z$. T. erhebliche Unterschiede. Während in den USA eine große Mehrheit der Arbeitgeber gewerkschaftlichen Aktivitäten negativ gegenüber steht, ist das zum Beispiel in Großbritannien teilweise anders (Heery/Simms 2007). Dort sind Organizing-Kampagnen in Betrieben signifikant erfolgreicher, in denen die Arbeitgeber keine explizit gewerkschaftsfeindliche Politik verfolgen. Entscheidend ist aber nicht, ob Kampagnen eher konfrontativ oder stärker kooperativ ausgerichtet sind. Vielmehr sind Gewerkschaften besonders erfolgreich, die ein großes Spektrum an Taktiken beherrschen und die jeweiligen OrganizingMethoden optimal auf das jeweilige Feld anzuwenden vermögen.

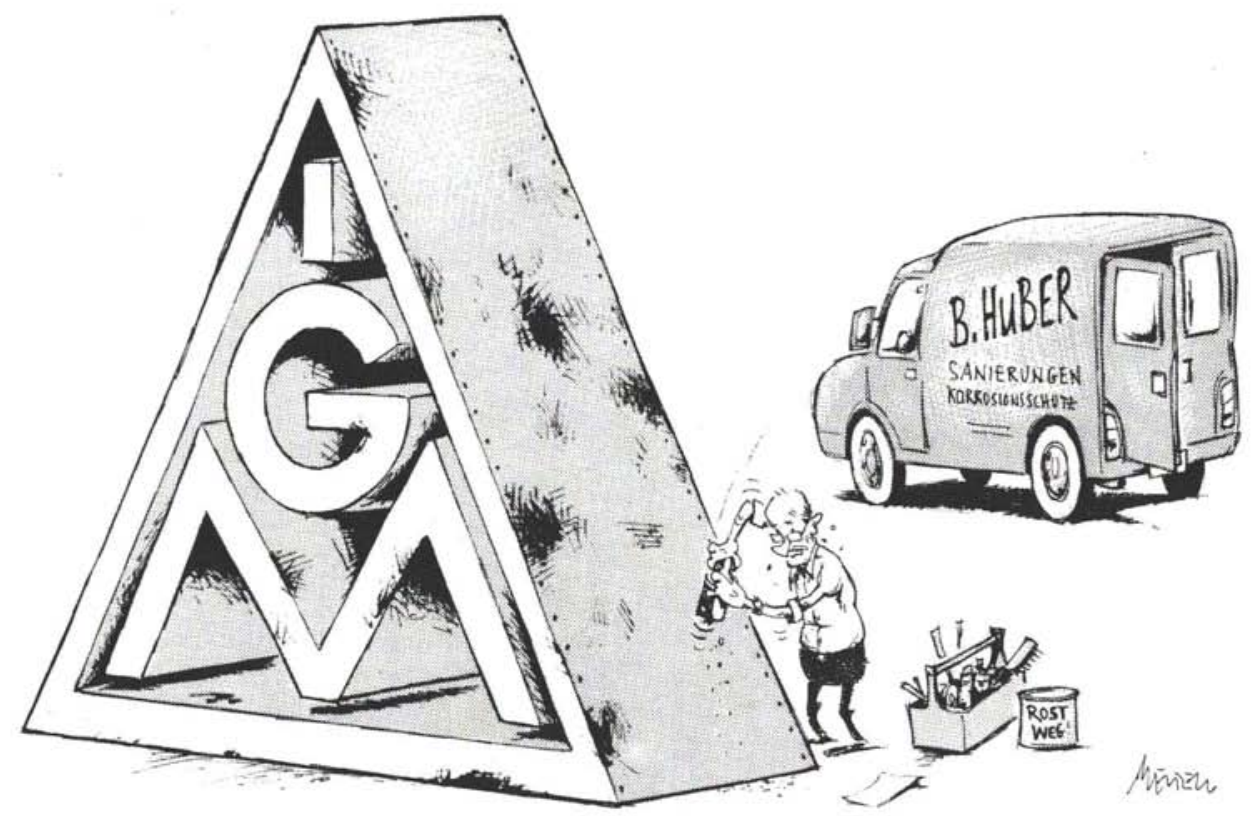




\section{Organizing - eine strategische Option für die deutschen Gewerk- schaften?}

Die Übertragbarkeit des US-amerikanischen Organizing-Ansatzes auf deutsche Verhältnisse wird kontrovers diskutiert. Carola Frege (2000) hat diese Frage angesichts der unterschiedlichen nationalen Systeme industrieller Beziehungen und der divergenten Gewerkschaftstraditionen vor Jahren mit einer gehörigen Portion Skepsis beantwortet. So habe Mitgliederwerbung in dem stark verrechtlichten deutschen Arbeitsbeziehungs-System bislang nur eine untergeordnete Rolle gespielt, faktisch sei die Aufgabe Betriebsräten übertragen worden. Wegen ihrer starken institutionellen Einbindung hätten die Gewerkschaften nur selten zum Mittel der Mitgliedermobilisierungen greifen müssen.

Ohne Zweifel hat Frege damit wichtige Punkte benannt, die gegen eine simple Übernahme von US-amerikanischen Organizing-Praktiken durch deutsche Gewerkschaftsgliederungen sprechen. Das gilt umso mehr, als die strategische Positionierung der DGB-Gewerkschaften noch immer eine deutlich andere ist als die der nordamerikanischen, Organizing-Gewerkschaften'. In den USA entspringt die starke Betonung von Bewegungsorientierung und des Coalition Building mit NGOs und Graswurzelinitiativen hauptsächlich der gewerkschaftlichen Schwäche in Betrieben und Unternehmen, sprich: der minimierten institutionellen Verhandlungsmacht. Aus diesem Grund macht es Sinn, die Durchsetzungsfähigkeit der Gewerkschaften mittels unkonventioneller Bündnispolitik erhöhen zu wollen. In Deutschland sind die Verhältnisse - noch - anders. Weder ist die Erosion institutioneller Verhandlungsmacht so weit fortgeschritten wie in den angelsächsischen Kapitalismen, noch sind soziale Bewegungen, NGOs und Graswurzelinitiativen derart stark, dass Bündnisse mit ihnen schwindende gewerkschaftliche Organisationsmacht kompensieren könnten. Auch aus diesem Grund scheuen die Gewerkschaften mehrheitlich davor zurück, Verhandlungsmacht, wie sie Mitbestimmung und Tarifsystem noch immer bieten, zugunsten einer riskanten Bündnispolitik mit oppositionellen Bewegungen zu opfern.

Allen berechtigten Warnungen vor unreflektierten Übernahmen von Praktiken aus anderen Arbeitsbeziehungsmodellen zum Trotz (Frege 2000) haben Organizing-Ansätze inzwischen auch in der Bundesrepublik rasch Karriere gemacht. Die Frage ist nicht mehr ob, sondern wie von den angelsächsischen Beispielen gelernt wird.

\section{Die organisationspolitische Falle}

Eine Triebkraft für rasches Organisationslernen ist der von Frege unterschätzte Druck auf die institutionalisierte Verhandlungsmacht der deutschen Gewerkschaften und der daraus resultierende Bedeutungsgewinn privilegierter Berufsgruppen mit hoher Primärmacht. Angesichts einer Konkurrenz, die auch mittels Prekarisierung von Beschäftigungsverhältnissen ausgetragen wird, tendieren Gruppen mit hoher Primärmacht wie die Lokführer oder die Ärzte dazu, ihre Interessen separat von anderen $\mathrm{Be}$ schäftigtengruppen wahrzunehmen. Anders als unternehmenskonforme, gelbe' Gewerkschaften zeichnen sich ihre nach absoluten Mitgliederzahlen eigentlich schwachen Organisationen durch hohe Lohn- und Gehaltsforderungen sowie hart geführte Konflikte aus. Die Erosion institutioneller (Tarif-)Macht der großen Mitgliedsgewerkschaften produziert so ein Revival separater Interessenkämpfe strukturell mächtiger Berufsgruppen. Parallel dazu wächst aber auch die Ungleichheit unter den Lohnabhängigen, weil vertretungsschwache Gruppen wie z. B. die ca. 22 Prozent Niedriglohnbezieher (Bosch/Weinkopf 2007) in dieser Konfliktkonstellation tendenziell den Kürzeren ziehen. Damit befinden sich die DGB-Gewerkschaften in einer Art Zwickmühle. Bleiben sie in der Fläche 
weniger durchsetzungsfähig, weil die alten korporativen Mechanismen nicht mehr funktionieren, drohen sie zwischen - teilweise schwach organisierten - Berufsgruppen mit großer struktureller Macht und dem wachsenden Heer prekär Beschäftigter eingeklemmt zu werden und weiter an Einfluss zu verlieren. Diese Konstellation ist es, die die Frage nach strategischen Alternativen aufwirft und das Interesse an Organizing-Praktiken weckt.

\section{Schritte zur Kampagnenorientierung}

Tatsächlich haben Lernprozesse längst begonnen, und sie machen sich auch in Organisationsbereichen bemerkbar, in denen eine bewusste Orientierung an Organizing-Ansätzen nicht vorhanden ist oder gar abgelehnt wird. Relativ unkompliziert erscheint auf den ersten Blick die Übernahme einzelner Instrumente des Organizing. So orientiert sich die LIDL-Kampagne von Verdi explizit am Vorbild einer Comprehensive Campaign (Schreieder 2007). Anders als im Falle der US-Gewerkschaften zielt die Kampagne jedoch zunächst auf Betriebsratsgründungen. Von der Etablierung betrieblicher Interessenvertreter erhofft sich die Gewerkschaft, allmählich auch die Beitrittsschwelle für die Beschäftigten des Unternehmens senken zu können. Im Vergleich zu den US-amerikanischen Vorbildern mangelt es der Kampagne allerdings erheblich an Ressourcen und an organisatorischer Verbindlichkeit.

Ein anderes Beispiel sind Kampagnen, die auf eine verbesserte Repräsentation und eine Rekrutierung prekär Beschäftigter zielen. Der inzwischen von mehreren Mitgliedsgewerkschaften getragenen Mindestlohn-Kampagne kommt hier eine symbolische und politische Funktion zu. Nicht minder wichtig sind dezentrale Initiativen. So haben zahlreiche DGB-Gliederungen mit Bestandsaufnahmen zur Entwicklung prekärer Beschäftigung begonnen. Im Organisationsbereich der IG Metall gibt es inzwischen mehrere Leiharbeitsprojekte, die darauf zielen, „Equal pay, equal treatment“ für Zeitarbeiter einzuklagen und Leiharbeiter in der Gewerkschaft zu organisieren Dabei werden sie von den Betriebsräten großer Unternehmen unterstützt. Noch fehlt es an geeigneten Beratungsund Organisationsstrukturen für potentielle Mitglieder, die räumlich verstreut arbeiten und häufig den Einsatzort wechseln. Dennoch beinhaltet dieser Ansatz ein Potential, das sich weit über das Leiharbeitsthema hinaus - zu einer Antidiskriminierungspolitik in Betrieben und Unternehmen ausbauen ließe. Interessant ist, dass punktuelle Organisierungserfolge dort möglich werden, wo die gewerkschaftlichen Protagonisten auf Formen der Selbstorganisation von Leiharbeitern setzen.

\section{Die Entdeckung der Mitglieder}

Damit ist bereits die politisch-organisatorische Form des Organizing, die Mitgliederpartizipation, berührt. Erstaunlich ist, dass sich im Kerngeschäft der deutschen Gewerkschaften, in der Betriebs- und Tarifpolitik, gravierende Veränderungen vollziehen. Gemeint ist die Neustrukturierung der Beziehungen von Betriebsräten, gewerkschaftlichen Funktionsträgern und (potenziellen) Mitgliedern, wie sie im Organisationsbereich der IG Metall teilweise erprobt wird. Angesichts der Legitimationsverluste, die betriebliche Konzessionspolitiken seit einiger Zeit verzeichnen (Rehder 2006), sind einzelne Bezirke und auch lokale Gliederungen der IG Metall dazu übergegangen, Standortvereinbarungen an Voten von Gewerkschaftsmitgliedern oder Belegschaften zu knüpfen. Selbst wenn direkte Partizipation niedrigschwellig, also auf dem Niveau von Belegschaftsbefragungen oder einer Beteiligung von Mitgliedern an der Aufstellung von Betriebsratslisten erfolgt, verändert sie die betrieblichen Machtspiele erheblich. Verhandlungen sind dann nicht mehr ausschließlich die Sache von Geschäftsleitungen, Betriebsräten und Gewerkschaftssekretären. Stattdessen tritt mit den Gewerkschaftsmitgliedern ein 
neuer kollektiver Akteur in Erscheinung, dessen Beteiligung an substanziellen Entscheidungen Ungewissheiten für die etablierten Betriebsund Tarifparteien mit sich bringt. Einmal nach ihrer Meinung gefragt, werden Gewerkschaftsmitglieder und Belegschaften derartige Beteiligungsmöglichkeiten auch künftig einklagen. Dennoch kann kein Zweifel bestehen, dass ganz im Sinne des Organizing-Modells - das Problem einer Stärkung gewerkschaftlicher Organisationsmacht innergewerkschaftlich, wie auch in der betrieblichen Arena, wieder an Bedeutung gewinnt.

In diesem Zusammenhang darf nicht übersehen werden, dass die deutschen Gewerkschaften den amerikanischen Organizing-Unions auf vielen betriebs- und tarifpolitischen Feldern noch immer ein gutes Stück voraus sind. Das gilt z. B. für arbeitspolitische Gestaltungsansätze, wie sie im Projekt, Gute Arbeit' zu unterschiedlichen Themen (Gesundheitsprävention, demographischer Wandel, Leistungssteuerung; Schröder/Urban 2009) erprobt werden. Diese Ansätze sind auch geeignet, um den Gewerkschaften in den expandierenden Segmenten mit qualifizierten, teilweise managementnahen Tätigkeiten Aufmerksamkeit zu verschaffen. Vorerst befinden sie sich aber noch im Stadium der Erprobung, und sie sind bislang nicht systematisch mit Initiativen zur Stärkung gewerkschaftlicher Organisationsmacht verbunden.

\section{Gewerkschaften und assoziierte Macht}

Am Maßstab einer Überwindung der gewerkschaftlichen Repräsentationskrise gemessen, handelt es sich bei den Organizing- und Erneuerungs-Ansätze sowohl in den USA als auch in Deutschland allenfalls um zarte Pflänzchen der Erneuerung, deren Wirkungen erst mit gebührendem zeitlichen Abstand bewertet werden können. Bislang fehlt es zudem an gesicherten wissenschaftlichen Erkenntnissen über die Re- sultate innovativer gewerkschaftlicher Praktiken. Als Besonderheit gegenüber der nordamerikanischen Kontrastfolie springt im Falle der deutschen Aktivitäten ins Auge, dass zahlreiche Aktivitäten auf eine Sensibilisierung vorhandener Betriebsräte oder eine Neugründung von Interessenvertretungen zielen. Auch aus diesem Grund schlagen sich Kampagnen und Initiativen nicht unmittelbar in Mitgliederzuwächsen nieder. Allerdings zeigt sich ebenfalls, dass Gewerkschaftsgliederungen sich durchaus als Schrittmacher einer aktiven Stärkung ihrer Organisationsmacht betätigen. Bis zu einer systematischen Ausrichtung nicht nur des Funktionärskörpers, sondern auch zumindest eines Teils der Mitgliedschaft auf offensives Organizing (Mitglieder werben Mitglieder) ist es aber noch ein weiter Weg.

Jenseits von Schwierigkeiten, die - auch aus institutioneller Divergenz resultieren, stehen Organizing-Ansätze in den USA wie auch in Deutschland vor einem gemeinsamen Problem. Bei allem gebotenen Pragmatismus ist zu klären, aus welchen Gründen sich Beschäftigte überhaupt in Gewerkschaften organisieren und engagieren sollen. Diese zentrale Sinnfrage lässt ein Organizing-Verständnis, das primär auf neue Methoden der Mitgliederrekrutierung abstellt, unbeantwortet. Dementsprechend fragil sind dann häufig Rekrutierungserfolge. Zwar kann es in aufwendigen Kampagnen im günstigsten Fall gelingen, rasch neue Mitglieder zu gewinnen, doch die Zugänge sind nicht nachhaltig. Sobald die betriebliche Routine wieder eintritt, Positionen und Funktionen vergeben sind, gehen neue Mitglieder häufig genauso rasch verloren wie sie gekommen sind. Im Fall der höchst innovativen - Leiharbeitskampagne der IG Metall (Holst u. a. 2009) könnte gegenwärtig eine solche Negativentwicklung drohen. Um organisationspolitisches Strohfeuer zu vermeiden, werden Organizing-Ansätze daher nicht umhinkommen, die Sinnfrage ihrer Adressaten zu beantworten. 


\section{Fazit}

Die Neujustierung des Verhältnisses von gewerkschaftlichen Funktionsträgern und Mitgliedern benötigt ein innovatives arbeitspolitisches Fundament. Generell lässt sich sagen, dass die finanzkapitalistisch dominierte $\mathrm{Re}$ strukturierung von Arbeit Demokratiefähigkeit schwächt (Schumann 2008). Leistungsintensivierung in den Bereichen qualifizierter Angestellten- und Informationsarbeit (Kratzer u. a. 2008), Retaylorisierungstendenzen in der Produktion und vor allem die Prekarisierung von Beschäftigungsverhältnissen rauben den Betroffenen die Energie und häufig auch die basale Sicherheit, die nötig ist, um an demokratischen Prozessen aktiv zu partizipieren. Gegen diesen Trend haben die Gewerkschaften mit dem Projekt, gute Arbeit' erfolgreich ein Thema in der politischen Öffentlichkeit platziert. Eine Erweiterung des Konzepts im Sinne demokratisch eingebetteter Arbeit drängt sich förmlich auf (Pickshaus; Urban 2009). So schwer es angesichts knapper Ressourcen auch sein mag: Organizing-Ansätze werden, sofern sie an einem Projekt demokratische Arbeit mitwirken wollen, expansiv, d. h. auf traditionell vertretungsschwache Gruppen ausgerichtet werden müssen. Faktisch geht es darum, das Prinzip ,equal pay, equal treatment“ für alle Gruppen zu realisieren, die unter „Überausbeutung“ und ständischer Diskriminierung leiden.

Soll das Ziel demokratische Arbeit überhaupt realistisch verfolgt werden, können gewerkschaftliche Interessenpolitiken nicht auf der Ebene der Arbeitsorganisation verharren. Sie werden dort ansetzen müssen, wo Unternehmen strategische Entscheidungen über die $\mathrm{Ba}$ lance von interner und externer Flexibilisierung (Leiharbeit, Werkverträge, Ausgründungen etc.) fällen. Organizing, eingebettet in eine Demokratisierungsstrategie, kommt letztlich ohne eine alternative Gesellschaftsvorstellung nicht aus.
Hier macht sich eine zentrale ideologische Schwäche vieler Arbeiterbewegungen in den Zentren bemerkbar. Über Jahrzehnte haben nicht nur die US-amerikanischen, sondern auch die deutschen Gewerkschaften auf die Formulierung eines alternativen Gesellschaftsprojekts verzichtet. In der Stunde der Systemkrise des Finanzmarkt-Kapitalismus macht sich dieser Mangel sträflich bemerkbar. Lange schien es, als sei das Beschwören der ,Systemfrage“ ein angestaubtes Relikt aus der Mottenkiste unverbesserlicher Traditionalisten. In einer historischen Konstellation, in der der FinanzmarktKapitalismus seine eigenen Legitimationsgrundlagen mit rapider Geschwindigkeit untergräbt und die Berater des neuen US-Präsidenten für langfristige Investitionen in einen ökologischsozialen ,New Deal' plädieren, wäre es fatal, würden sich die Gewerkschaften mit organisationspolitischem Pragmatismus zufrieden geben. Gerade die aktiven Gruppen benötigen eine Grundüberzeugung, auf deren Basis sie die gesellschaftlichen Krisenphänomene in Argumente für gewerkschaftliche Organisierung ummünzen können. Die Idee, mit OrganizingAnsätzen demokratische Verhältnisse zu fördern, könnte Teil einer solchen Grundüberzeugung sein.

Dass die Gewerkschaften einen Erneuerungsprozess, der sie in der Perspektive - wieder - zu Trägern eines alternativen Gesellschaftsprojekts machen könnte, allein aus sich selbst heraus initiieren, ist indessen sowohl in den USA als auch in Deutschland unwahrscheinlich. Ohne Herausforderungen durch soziale Bewegungen und Unterstützung aus dem politischen Raum, dürfte die Revitalisierung von Gewerkschaftsbewegungen wohl kaum gelingen.

Man darf gespannt sein, ob der Funke, den gewerkschaftliche Revitalisierung durch Lernen von sozialen Bewegungen ausgelöst hat, von Übersee nach Europa und Deutschland überspringen wird. Trotz mancher Gemeinsamkei- 
ten mit Organizing-Ansätzen von US-Gewerkschaften haben sich innovative Praktiken auch in Deutschland bislang nicht zu einer kohärenten Interessenpolitik verdichten können. Es fehlt an einer geeigneten organisatorisch-politischen Infrastruktur, an Organizing-Akademien, an politischer Weiterbildung innerhalb der Gewerkschaften, an spezifischen Qualifizierungsangeboten, aber auch an geeigneten Kooperationsstrukturen mit Wissenschaftlern, Journalisten und Medienfachleuten. Funktionsträger, die bereit und in der Lage wären, ihren Aktivitätsschwerpunkt jenseits der Organisationsroutinen zu setzen, um aktiv in betriebliche OrganizingProzesse und gesellschaftliche Konflikte einzugreifen, sind noch rar gesät. Von einem wirklichen strategischen Organisationswandel, wie ihn ein politisches Organizing-Verständnis impliziert, sind die DGB-Gewerkschaften vorerst noch weit entfernt. Punktuell sind die Praktiker bei der Rekonstruktion gewerkschaftlicher Organisationsmacht in Betrieben aber schon weiter, als es der grassierende Niedergangsfatalismus Glauben machen will. Auch und gerade für diese Ansätze gilt, dass sie nicht das Problem, sondern Teil der Lösung sind.

Klaus Dörre ist Professor für Arbeits-, Industrie-und Wirtschaftssoziologie an der Friedrich-Schiller Universität Jena. Kontakt: klaus.doerre@uni-jena.de.

Oliver Nachtwey ist zurzeit Vertretungsprofessor der Universität Göttingen und wissenschaftlicher Mitarbeiter an der Uni Jena. Kontakt: oliver.nachtwey@uni-jena.de.

\section{Literatur}

Aronowitz, Stanley 2005: On the Future of American Labor. In: Working USA. The Journal of Labor and Society 8 (3), S. 271-291.

Beaud, Stephane/Pialoux, Michel 2004: Die verlorene Zukunft der Arbeiter. Konstanz: UVK Universitätsverlag.
Bosch, Gerhard/Weinkopf, Claudia (Hg.) 2007: Arbeiten für weniger Geld. Niedriglohnbeschäftigung in Deutschland. Frankfurt/M: Campus.

Brinkmann, Ullrich/Choi, Hea-Lee/Detje, Richard/Dörre, Klaus/Holst, Hajo/Karakayali, Serhat/Schmalstieg, Chatarina 2008: Strategic Unionism. Aus der Krise zur Erneuerung? Wiesbaden: VS-Verlag.

Bronfenbrenner, Kate/Hickey, Rob 2004: Changing to Organize: A National Assessment of Union Organizing Strategies, in: Milkman/ Voss (Hg.), a.a.O.: 17-61.

Clawson, Dan 2005: Organizing, Movements, and Social Capital. In: Labor Studies Journal 29 (4), S. 37-44.

Cregan, Christina 2005: Can organizing work? An inductive analysis of individual attitudes toward union membership. In: Industrial and Labor Relations Review 58 (2), S. 282304.

Crosby, Michael 2005: Power at Work. Rebuilding the Australian Union Movement. Sydney, The Federation Press.

Dörre, Klaus/Röttger, Bernd 2006: Im Schatten der Globalisierung. Strukturpolitik, Netzwerke und Gewerkschaften in altindustriellen Regionen. Wiesbaden, VS-Verlag.

Dribbusch, Heiner 2007: Das OrganizingModell. Entwicklung, Varianten, Umsetzung, in: Bremme u.a. (Hg.), a.a.O.: 24-52.

Dribbusch, Heiner 2003: Gewerkschaftliche Mitgliedergewinnung im Dienstleistungssektor. Ein Drei-Länder-Vergleich im Einzelhandel. Berlin, Edition Sigma.

Fantasia, Rick/Voss, Kim 2004: Hard Word. Remaking the American Labor Movement. Berkeley, University of California Press.

Fiorito, Jack 2004: Union Renewal and the Organizing Model in the United Kingdom. In: Labor Studies Journal 29 (2), S. 21-53.

Frege, Carola M. 2000: Gewerkschaftsreformen in den USA. Eine kritische Analyse des 
,Organisierungsmodells‘. In: Industrielle Beziehungen 7 (3), S. 260-280.

Frege, Carola M./Kelly, John 2004: Union Revitalisation Strategies in Comparative Perspective. European Industrial Relations Journal, (9) 1, S. 7-24.

Frege, Carola M./Kelly, John 2004: Varieties of Unionism. Strategies for Union Revitalization in a Globalizing Economy. Oxford, University Press.

Gall, Gregor 2005: Organizing Non-Union Workers as Trade Unionists in the ,New Economy ' in Britain. In: Economic and Industrial Democracy 26 (1), S. 41-63.

Heery, Edmund/Simms, Melanie 2007: Employer Responses to Union organising in the United Kingdom, Manuskript, Warwick.

Holst, Hajo/Nachtwey, Oliver/Dörre, Klaus (2009): Funktionswandel von Leiharbeit. Neue Nutzungsstrategien und ihre arbeits- und mitbestimmungspolitischenpolitischen Folgen. OBS-Arbeitsheft 61, Frankfurt a. M.

Kratzer, Nick/Menz, Wolfgang/Nies, Sarah/ Sauer, Dieter (2008): Leistungspolitik als Feld umkämpfter Arbeit. In: Prokla, Heft 150, 38. Jg., S. 11-26.
Urban, Hans-Jürgen 2009: Gute Arbeit als Strategie. Perspektiven gewerkschaftlicher Arbeitspolitik. Erscheint in: Schröder, Lothar/Urban, Hans-Jürgen (Hg.): Gute Arbeit, a.a.O: 1926.

Rehder, Britta 2006: Legitimitätsdefizite des Co-Management. Betriebliche Bündnisse für Arbeit als Konfliktfeld zwischen Arbeitnehmern und betrieblicher Interessenvertretung. In: Zeitschrift für Soziologie 3, S. 227-242.

Schreieder, Agnes 2007: Die LIDL-Kampagne - ein Zukunftsmodell der Gewerkschaften. In: Bremme u.a. (Hg.), a.a.O.: S. 153-174.

Schröder, Lothar/Urban, Hans-Jürgen (Hg.) 2009: Gute Arbeit. Handlungsfelder für Betriebe, Politik und Gewerkschaften. Frankfurt/M., Bund-Verlag.

Schumann, Michael 2008: Kampf um Rationalisierung - Suche nach neuer Übersichtlichkeit. In: WSI-Mitteilungen 2/2008, S. 379-386.

Silver, Beverly 2005: Forces of Labour. Arbeiterbewegung und Globalisierung seit 1870 . Berlin, Assoziation A-Verlag.

Voss, Kim/Sherman, Rachel 2000: Breaking the Iron Law of Oligarchy. Union Revitalization in the American Labor Movement. In: American Journal of Sociology 106 (2), S. 303-349. 